\title{
Prognostic Evaluation of Mortality after Pediatric Resuscitation Assisted by Extracorporeal Life Support
}

\author{
Aurélie De Mul ${ }^{1}$ Duy-Anh Nguyen ${ }^{1} \quad$ Carsten Doell $^{2,3}$ Marie-Hélène Perez ${ }^{4}$ Vincenzo Cannizzaro ${ }^{2,3}$ \\ Oliver Karam ${ }^{1,5}$
}
1 Pediatric Intensive Care Unit, Geneva University Hospital, Geneva, Switzerland
2 Department of Intensive Care Medicine and Neonatology, University Children's Hospital of Zurich, Zurich, Switzerland
${ }^{3}$ Children's Research Center, University Children's Hospital of Zurich, University of Zurich, Zurich, Switzerland
${ }^{4}$ Pediatric Intensive Care Unit, Lausanne University Hospital,
Lausanne, Switzerland
${ }^{5}$ Division of Pediatric Critical Care, Children's Hospital of Richmond at VCU, Richmond, VA, United States

J Pediatr Intensive Care 2019;8:57-63.

\begin{abstract}
Address for correspondence Oliver Karam, MD, PhD, Division of Pediatric Critical Care Medicine, Children's Hospital of Richmond at VCU, 1250 East Marshall Street, Richmond, VA 23298, United States (e-mail: oliver.karam@vcuhealth.org).
\end{abstract}

\begin{abstract}
Keywords

- extracorporeal life support

- extracorporeal membrane oxygenation

- cardiopulmonary resuscitation

- prognostic factors

- children

To improve survival rates during cardiopulmonary resuscitation (CPR), some patients are put on extracorporeal life support (ECLS) during active resuscitation (ECPR). Our objective was to assess the clinical outcomes after pediatric ECPR in Switzerland and to determine pre-ECPR prognostic factors for mortality. The present study is a retrospective analysis. The study setting included three pediatric intensive care units in Switzerland that use ECPR. All patients ( $<16$ years old) undergoing ECPR from 2008 to 2016 were included in the study. There were no interventions. Data before ECLS initiation and clinical outcomes were collected. An ECPR score was designed to predict mortality, based on variables significantly different between survivors and non-survivors. Fifty-five patients were included, with a median age of 13.5 months. Eighty percent were cardiac patients. The mortality rate was $75 \%$. Mortality was significantly associated with CPR duration $(p=0.02)$, last lactate $(p=0.05)$, and last $\mathrm{pH}(p=0.01)$ before ECLS initiation. Based on these three variables, an ECPR score was designed as follows: CPR duration (in minutes): 1 point if $<40 ; 2$ points if $\geq 40 ; 3$ points if $\geq 60 ; 6$ points if $\geq 105$. Lactate (in $\mathrm{mmol} / \mathrm{L}$ ): 1 point if $<8 ; 2$ points if $\geq 8 ; 3$ points if $\geq 14 ; 6$ points if $\geq 18$. $\mathrm{pH}: 1$ point if $>7.00 ; 2$ points if $\leq 7.00 ; 3$ points if $\leq 6.85 ; 6$ points if $\leq 6.60$. The area under the receiver-operating characteristic curve was 0.74 . The positive predictive value of a score $\geq 9$ was $94 \%$. In our population, a score based on three variables easily available prior to ECLS initiation had good discrimination and could appropriately predict mortality. This score now needs validation in a larger population.
\end{abstract}

\section{Introduction}

Extracorporeal cardiopulmonary resuscitation (ECPR) allows for adequate tissue perfusion in certain situations of refractory cardiac arrest, when prolonged conventional cardiopulmonary resuscitation (CCPR) failed to restore spontaneous circulation. The first reports of pediatric ECPR date back to the 1980 s. $^{1}$
A large pediatric ECPR study including 3756 patients showed greater survival and better neurological outcome with ECPR compared with CCPR ( 40 vs. $27 \%$ and 27 vs. $18 \%$, respectively). ${ }^{2}$ The Extracorporeal Life Support Organization (ELSO) Registry reports an increased number of ECPR patients by $67 \%$ from 2009 to $2015 .^{3}$ The only international guidelines currently in received

April 13, 2018

accepted after revision

June 3, 2018

published online

July 11,2018
Copyright @ 2019 by Georg Thieme

Verlag KG, Stuttgart . New York
DOI https://doi.org/

10.1055/s-0038-1667012. ISSN $2146-4618$. 
use were published by the International Liaison Committee on Resuscitation in 2015. It suggests considering ECPR for cardiac diagnoses who have in-hospital cardiac arrest. ${ }^{4}$ Identifying the best candidates for pediatric ECPR is mandatory. Indeed, despite its wide acceptance and use, ECPR is an invasive mechanical support that is associated with an important morbidity and mortality due to extracorporeal membrane oxygenation (ECMO)-related complications. Moreover, depending on the patient's neurological prognosis, its use might be futile, and even harmful. Lastly, this complex technique requires substantial financial and human resources.

Aiming to improve patient selection, some authors identified prognostic factors for mortality. Joffe et al reviewed factors predicting mortality in 17 studies including 762 pediatric patients with ECPR between 2000 and 2011. ${ }^{5}$ The most significant factors were a noncardiac disease leading to cardiac arrest, an acute kidney failure during extracorporeal life support (ECLS), neurological complications during ECLS, and a very low $\mathrm{pH}$ during ECLS. Unfortunately, these factors are not helpful to decide whether or not ECPR should be initiated, as most of these risk factors can only be assessed after ECMO initiation.

The aim of our study is to report ECPR clinical outcomes in three Swiss tertiary pediatric intensive care units (PICUs), to identify factors associated with mortality and to establish a prognostic score for mortality, using variables that are available prior to ECLS initiation.

\section{Materials and Methods}

This report describes a retrospective study performed in three tertiary PICUs in Geneva, Lausanne, and Zurich, Switzerland, from January 2008 to December 2016. We included all consecutive children ( $<16$ years old) at these three institutions who underwent ECPR; no exclusion criteria were utilized in this study, and each qualifying patient was included. ECPR is defined, according to ELSO, as ECMO cannulation during CPR with ongoing chest compressions.

This study was approved by the ethics review board "Commission Cantonale d'Ethique de la Recherche," which waived the need for individual consent.

\section{Outcomes}

The primary outcome was in-hospital mortality after ECLS initiation. The secondary outcome was neurological status of hospital survivors at discharge, as assessed by the Pediatric Cerebral Performance Category (PCPC) score. ${ }^{6}$ The PCPC score evaluates cognitive impairment. It has six categories: (1) normal outcome, (2) mild disability (regular school, but grades perhaps not age-appropriate), (3) moderate disability (ageappropriate independent activities of daily life but special education classroom and/or learning deficit present), (4) severe disability (dependent on others for daily support because of impaired brain function), (5) coma or vegetative state (without interaction with the environment); and (6) brain death. This assessment was systematically performed by a trained physician for every patient who had a history of cardiac arrest. We recorded demographic data, diagnosis at
PICU admission, presence of comorbidities, suspected etiology of circulatory arrest, last laboratory tests before ECLS initiation, length of CPR until ECLS initiation, central or peripheral ECLS cannula insertion site, ECLS duration, length of PICU stay, and, when applicable, time and cause of death. Comorbidities were defined as any condition associated with organ dysfunction that required support in a critical care setting. ECLS initiation is defined as the time between circulatory arrest and oxygenated blood flow returned to the patient through the ECLS circuit.

For patients with two ECPR events, only the second run was used to evaluate the mortality outcome.

\section{Extracorporeal Life Support Programs}

Geneva's PICU has an average of 150 postoperative cardiac patients per year over the past 9 years and an average of eight ECLS runs per year over the past 5 years. Its ECLS program was initiated in 1996. Lausanne's PICU has an average of 150 postoperative cardiac patients per year as well. Their first ECLS occurred in 2011, but the ECLS program formally began in 2015. Zurich's PICU has an average of 240 postoperative cardiac surgery patients per year. Zurich's ECLS program was initiated in 2004 with an average of nine ECLS runs per year over the 9-year study period. An average of 15 ECLS runs per year were performed in the past 5 years.

In all three centers, ECLS cannulation is always performed by cardiac surgeons in collaboration with specialized perfusion technicians, none of which are in-house during nights and weekends. There is no formal ECPR recommendation in place. Hypothermia is not used after cardiac arrest, but hyperthermia is actively avoided in these patients.

\section{Statistical Analysis}

Descriptive statistics are reported as mean \pm standard deviation, median (interquartile range, IQR), or proportions with their 95\% confidence intervals (CIs). Due to the small sample size, nonparametric tests were used to evaluate the association between pre-ECLS initiation variables and survival status: Mann-Whitney $U$ test for continuous variables and Fisher's exact test for dichotomous variables. All tests were two-sided, with an $\alpha$ level of 0.05 . The power to detect an area under the receiver-operating characteristic curve (AUC) of 0.75 with an $\alpha$ of 0.05 and an allocation ratio of 2.92:1 (41 non-survivors, 14 survivors) was $90 \%$.

\section{Design and Testing of the Score}

We used the following strategy to design the ECPR score to predict mortality. All variables that were significantly associated with mortality were used for the score after demonstrating the absence of colinearity. Three cutoffs, delimiting four categories, were identified for each variable in the score based on the 25th, 50th, and 75th percentile of the values in the nonsurviving group. The coefficients for each category were based on the individual risk ratios of mortality for that category compared with the next. The final coefficients were based on the average risk for all three variables and were within the 95\% $\mathrm{Cl}$ of the risk ratio, to have a homogenous and simple score. Of note, it was not possible to use a logistic regression model to design the score due to the small sample size. 
The performance of the score was evaluated as follows: Discrimination, which refers to the ability of the score to separate non-survivors from survivors across the whole group, ${ }^{7}$ was evaluated using the area under the receiveroperating characteristic curve with its $95 \% \mathrm{CI}$. The sensitivity analysis was performed with a Youden's J statistic calculation.

Calibration was assessed by directly comparing the observed and customized predicted mortality across subcategories of risk. We employed the Hosmer-Lemeshow goodness-of-fit test, where a $p$ value $>0.05$ indicates acceptable calibration. ${ }^{8}$

All statistical analyses were performed with SPSS version 24 for Mac (IBM, Armonk, New York, United States).

\section{Results}

\section{Demographic Data}

Over 9 years, 55 patients were treated with 56 ECPR (one patient required two ECPRs and died after the second run). Sixty-four percent (35/55) were male. The median weight was $7.55 \mathrm{~kg}$ (IQR 4.25-20.0) and the median age 13.5 months (IQR 1-69). As showed in - Fig. 1, 80\% (45/55) of the patients had cardiac conditions leading to circulatory arrest. The 34 congenital heart defects were physiologically univentricular heart $(n=8)$, conotruncal heart defect $(n=8)$, transposition of the great arteries $(n=7)$, heterotaxy anomaly $(n=4)$, valvulopathy $(n=4)$, and ventricular septal defect $(n=3)$. The median number of circulatory arrests prior to ECLS initiation was 1 (IQR 1-2). The median length of CPR prior to ECLS initiation was 60 minutes (IQR 40-90).

\section{Extracorporeal Life Support Run and Outcome}

The median length of ECLS was 105 hours (IQR 52-162). Cannulation was central for $2 / 3$ patients in Lausanne, 12/22 in Geneva, and $26 / 27$ in Zurich $(p=0.002)$. Central cannulation was performed in $71 \%(10 / 14)$ of the survivors versus $79 \%$ (32/41) of the non-survivors $(p=0.71)$.

The overall hospital mortality rate was 75\% (41/55). Sixtyfour percent (26/41) died of multiple organ failure, 34\% (14/41) of brain death or devastating hypoxic ischemic encephalopathy, and 2\% (1/41) of hemorrhagic shock. Seventy-three percent (30/41) of the patients died while on ECLS, as a consequence of multiple organ failure $(n=16)$, brain death $(n=7)$, severe anoxic-ischemic encephalopathy $(n=4)$, refractory heart failure $(n=1)$, refractory hemorrhagic shock $(n=1)$, and sepsis $(n=1)$.

Twenty-seven percent (11/41) of the patients died after ECLS decannulation after a median of 12 days (IQR 1-30). Fifty-five percent (6/11) died of multiple organ failure and $45 \%$ (4/11) died after redirection of care, due to severe anoxic-ischemic encephalopathy.

Fourteen patients (25\%) survived ECPR and were discharged alive. Seven patients (50\% of survivors, $13 \%$ of all patients) had no neurological deficit, as defined by a PCPC score of 1 . Two patients ( $14 \%$ of survivors) had mild disabilities (PCPC score of 2), whereas four patients (28\% of survivors) had moderate disabilities (PCPC score of 3), and one patient (7\% of survivors) had severe disabilities (PCPC score of 4).

There was no significant change in the mortality rate from 2008 to 2016 ( $p=0.28)$.

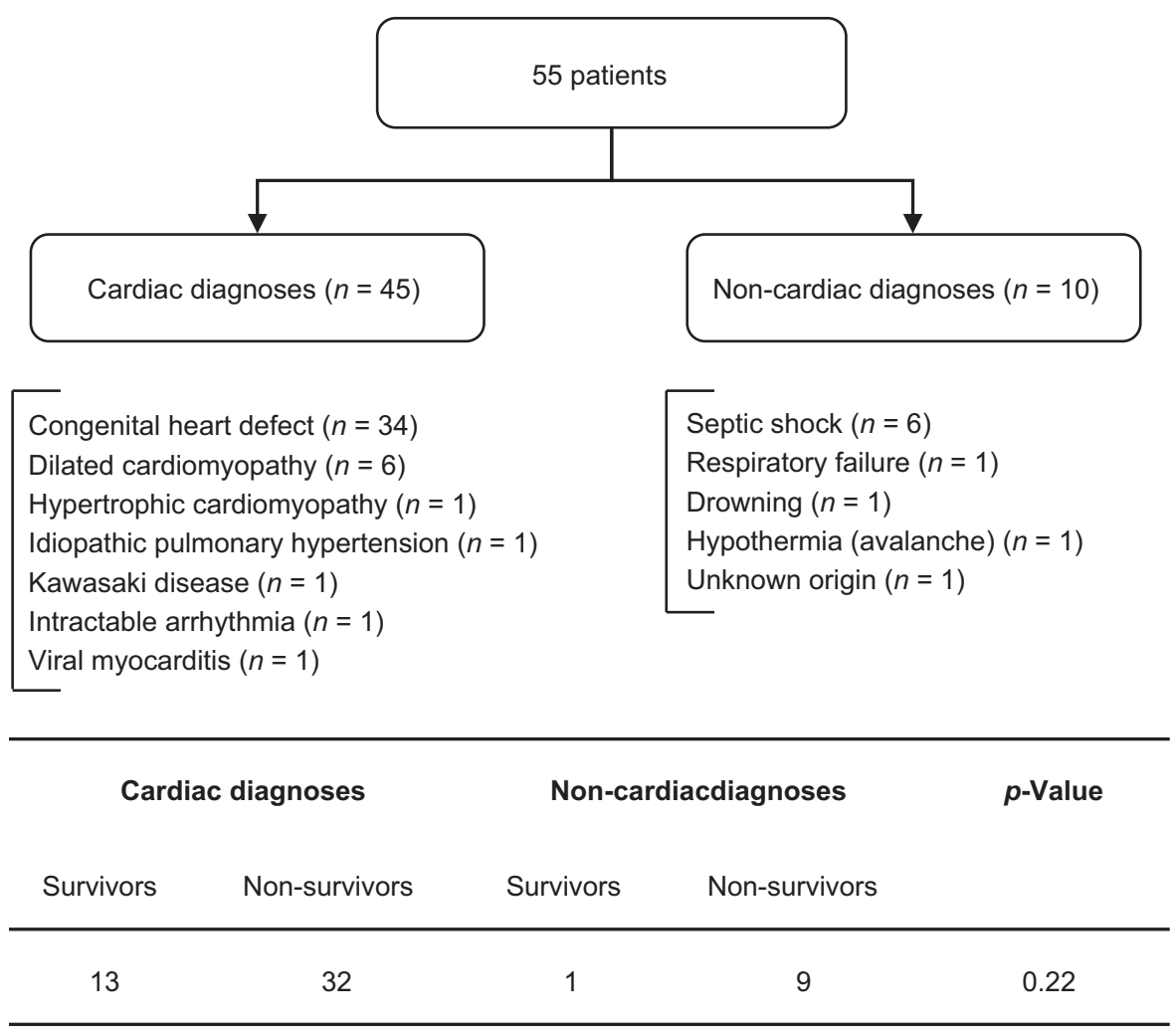

Fig. 1 Flow chart of the population, describing the patients with cardiac diagnoses and those with noncardiac diagnoses. The table below the flowchart describes the outcome between these two subgroups. 
Table 1 Variables available prior to extracorporeal life support initiation

\begin{tabular}{|l|l|l|l|l|}
\hline Variables & $\begin{array}{l}\text { Number of patients in } \\
\text { which it was measured }\end{array}$ & $\begin{array}{l}\text { Survivors } \\
\mathbf{n = 1 4}\end{array}$ & $\begin{array}{l}\text { Non-survivors } \\
\boldsymbol{n}=\mathbf{4 1}\end{array}$ & $p$-Value \\
\hline Age (years) & 55 & $1.4(0.4-8)$ & $1.0(0.1-5.4)$ & 0.47 \\
\hline Weight $(\mathrm{kg})$ & 55 & $10.0(5.6-26.9)$ & $7.3(3.9-19.5)$ & 0.22 \\
\hline Underlying cardiac condition & 55 & $12(86 \%)$ & $31(76 \%)$ & 0.71 \\
\hline Comorbidities & 55 & $4(29 \%)$ & $14(27 \%)$ & 0.9 \\
\hline Length of CPR (minutes) & 52 & $45(18-49)$ & $60(40-105)$ & 0.02 \\
\hline Last lactate (mmo/L) & 50 & $11.1(5.5-14.2)$ & $13.9(8.1-17.5)$ & 0.05 \\
\hline Last potassium (mEq/L) & 51 & $4.6(4.1-5.3)$ & $5.2(4.2-6.8)$ & 0.17 \\
\hline Last pH & 50 & $7.06(6.9-7.16)$ & $6.85(6.58-7.02)$ & 0.01 \\
\hline Last pCO (torr) & 50 & $56(44-77)$ & $71(44-105)$ & 0.48 \\
\hline Last bicarbonate $(\mathrm{mmol} / \mathrm{L})$ & 50 & $12.2(9.6-18.5)$ & $11.4(5.8-17.7)$ & 0.27 \\
\hline Absent pupillary reflex & 11 & $1(7 \%)$ & $3(7 \%)$ & 0.36 \\
\hline
\end{tabular}

Abbreviations: CPR, cardiopulmonary resuscitation; ECLS, extracorporeal life support; IQR, interquartile range; $\mathrm{pCO}_{2}$, partial pressure of carbon dioxide. Values are presented as either median and IQR or number and proportion. Laboratory values were the last measured prior to ECLS initiation.

\section{Risk Factor for Mortality}

The variables measured prior to ECLS initiation are available in - Table 1.

Mortality was significantly associated with length of CPR before ECLS initiation, last lactate, and last $\mathrm{pH}$ before ECLS initiation ( $p=0.02, p=0.05$, and $p=0.01$, respectively). There was a poor correlation between $\mathrm{pH}$ and lactates (Pearson $R^{2}=0.09$ ). The presence of comorbidities and underlying cardiac conditions were not associated with worse outcomes after $\operatorname{ECPR}$ ( $p=0.9$ and $p=0.71$, respectively).

\section{Score to Predict Mortality after ECPR}

Based on these three variables, we designed an ECPR score. The thresholds for each variable were based on the quartiles in the non-survivors. The coefficient of each category was based on the relative risk of mortality compared with baseline $(-$ Table 2$)$. The final score is presented in $\boldsymbol{- T}$ Table $\mathbf{3}$. The score ranges from 3 to 18 points.

Each incremental point increased the risk of mortality by $39 \%$ $(p=0.01)$. Using a cutoff of $\geq 9$, the sensitivity, specificity, and positive predictive value were $41 \%(95 \% \mathrm{Cl}$ : $26-58), 93 \%$ (95\% Cl: 66-100), and 94\% (95\% CI: 71-99), respectively (-Fig. 2).

\section{Discrimination and Calibration of the Score}

The AUC was 0.74 (95\% CI: 0.61-0.87). The Hosmer-Lemeshow chi-square value was $4.33(p=0.63)$.

\section{Discussion}

In this study, we found a significant association between mortality and length of CPR, last lactate, and last $\mathrm{pH}$ before ECLS initiation. We used those variables, easily available prior to ECLS initiation, to design a score to predict mortality.

The parameters that are used in this score have a sound physiological basis. Serum lactate and pH before ECLS initiation reflect the severity of tissue hypoxemia and are an indicator of oxygen delivery and hence CPR quality, although they might also reflect renal or liver failure. The association between lactate and $\mathrm{pH}$ with mortality in our study is in accordance with other previous pediatric and adult findings. ${ }^{5,9,10}$ Thus, in a pediatric series of $695 \mathrm{ECPR}$, the risk of mortality was halved when the pre-ECMO pH was above $7.17 .^{11}$ Recently, a large adult meta-analysis including 841 ECPR showed that survivors had significantly

Table 2 Univariate risk ratio and 95\% Cl of mortality, for the second, third, and fourth category in reference to the first category. The average risk for the three variables is presented on the last line

\begin{tabular}{|l|l|l|l|l|}
\hline Variables & 1st Quartile & 2nd Quartile & 3rd Quartile & 4th Quartile \\
\hline Length of CPR (minutes) & Ref & $1.0(0.2-4.5)$ & $4.6(0.7-30.4)$ & 7.1 (0.6-75.1) \\
\hline Lactate (mmol/L) & Ref & $1.4(0.3-6.4)$ & $1.9(0.3-10.4)$ & No survivor \\
\hline $\mathrm{pH}$ & Ref & $4.1(0.7-24.0)$ & $3.6(0.6-21.6)$ & No survivor \\
\hline Average risk & 1 & 2.2 & 3.4 & 7.1 \\
\hline
\end{tabular}

Abbreviations: $\mathrm{Cl}$, confidence interval; $\mathrm{CPR}$, cardiopulmonary resuscitation; Ref, reference.

Note: The different quartiles are $<40,40-59,60-104$, and $\geq 105$ for length of CPR; $<8.0,8.0-13.9,14.0-17.9$, and $\geq 18.0$ for lactates; and $>7.0,6.85-7.0,6.61-6.84$, and $\leq 6.60$ for $\mathrm{pH}$, respectively.

For example, a patient with a lactate of $14.0 \mathrm{mmol} / \mathrm{L}$ (in the third quartile) has a 1.9-fold increase in the risk of mortality, as compared with a patient with a lactate of $7.0 \mathrm{mmol} / \mathrm{L}$. 
Table 3 Score to predict mortality after extracorporeal life support during active resuscitation

\begin{tabular}{|l|l|l|l|l|}
\hline Variables & $\mathbf{1}$ Point & 2 Points & 3 Points & 6 Points \\
\hline $\begin{array}{l}\text { Length of } \\
\text { CPR } \\
\text { (minutes) }\end{array}$ & $<40$ & $40-59$ & $60-104$ & $\geq 105$ \\
\hline $\begin{array}{l}\text { Lactate } \\
(\mathrm{mmol} / \mathrm{L})\end{array}$ & $<8.0$ & $8.0-13.9$ & $14.0-17.9$ & $\geq 18.0$ \\
\hline $\mathrm{pH}$ & $>7.00$ & $6.85-7.00$ & $6.61-6.84$ & $\leq 6.60$ \\
\hline
\end{tabular}

Abbreviations: CPR, cardiopulmonary resuscitation; ECLS, extracorporeal life support.

Note: If a variable was not measured prior to ECLS initiation, it was considered to be normal.

lower serum lactate concentration $(-3.53 \mathrm{mmol} / \mathrm{L})$ than non-survivors. ${ }^{12}$

We also found a significant association between length of CPR before ECLS initiation and mortality. The effect of CPR duration is debated. Some authors have suggested that there was no association between CPR duration before ECLS initiation and mortality. ${ }^{13-16}$

In our series, prolonged CPR duration might be due to a few factors. For example, some out-of-hospital cardiac arrest cases were put on ECMO and were hence included in the study; the absence of in-house ECMO teams during nights and weekends might potentially have prolonged CPR time; and some attendings might have beliefs that prolonged CPR time does not always lead to unfavorable outcome. For example, we report a patient with grossly intact neurological status at the time of discharge despite prolonged CPR duration up to 120 minutes. Other case series and case reports have reported ECPR survivors with favorable neurological outcome after CPR length up to 220 minutes. ${ }^{17-19}$ On the other hand, Matos et al published a large prospective and multicenter study, which included 3,419 children with cardiac arrest between 2000 and 2009. ${ }^{20}$ They showed that when CRP lasted between 1 and 15 minutes, survival decreased by $2.1 \%$ per minute and favorable neurological outcomes decreased by $1.2 \%$ per minute. Adjusted probability of survival was $41 \%$ for CPR duration of 1 to 15 minutes and $12 \%$ for more than 35 minutes. A meta-analysis including 288 pediatric patients with ECPR showed that mortality was doubled when the duration of CPR was longer than 30 minutes (odds ratio 2.1). ${ }^{21}$ The previously cited large adult meta-analysis by Debaty et al revealed that a shorter CPR duration before ECLS was significantly associated with survival. ${ }^{12}$ Other recent pediatric and adult population studies support these data. ${ }^{9,22-25}$ Therefore, it seems biologically plausible that increased length of CPR before ECLS initiation is associated with worse clinical outcome, especially in conjunction with markers of poor end-organ perfusion indicating insufficient oxygen delivery.

The score, which was derived from variables available before ECLS initiation, accurately predicted mortality after ECLS initiation. The AUC of 0.74 , considered as good, ${ }^{8}$ is comparable to other clinical scores reported in the ELSO registry. ${ }^{26}$ Using a cutoff of 9 , this score has a positive predictive value of $94 \%$. This could aid clinicians when making the final decision to initiate ECLS and perhaps avoid futile treatment, especially in the centers without a 24/7 in-house

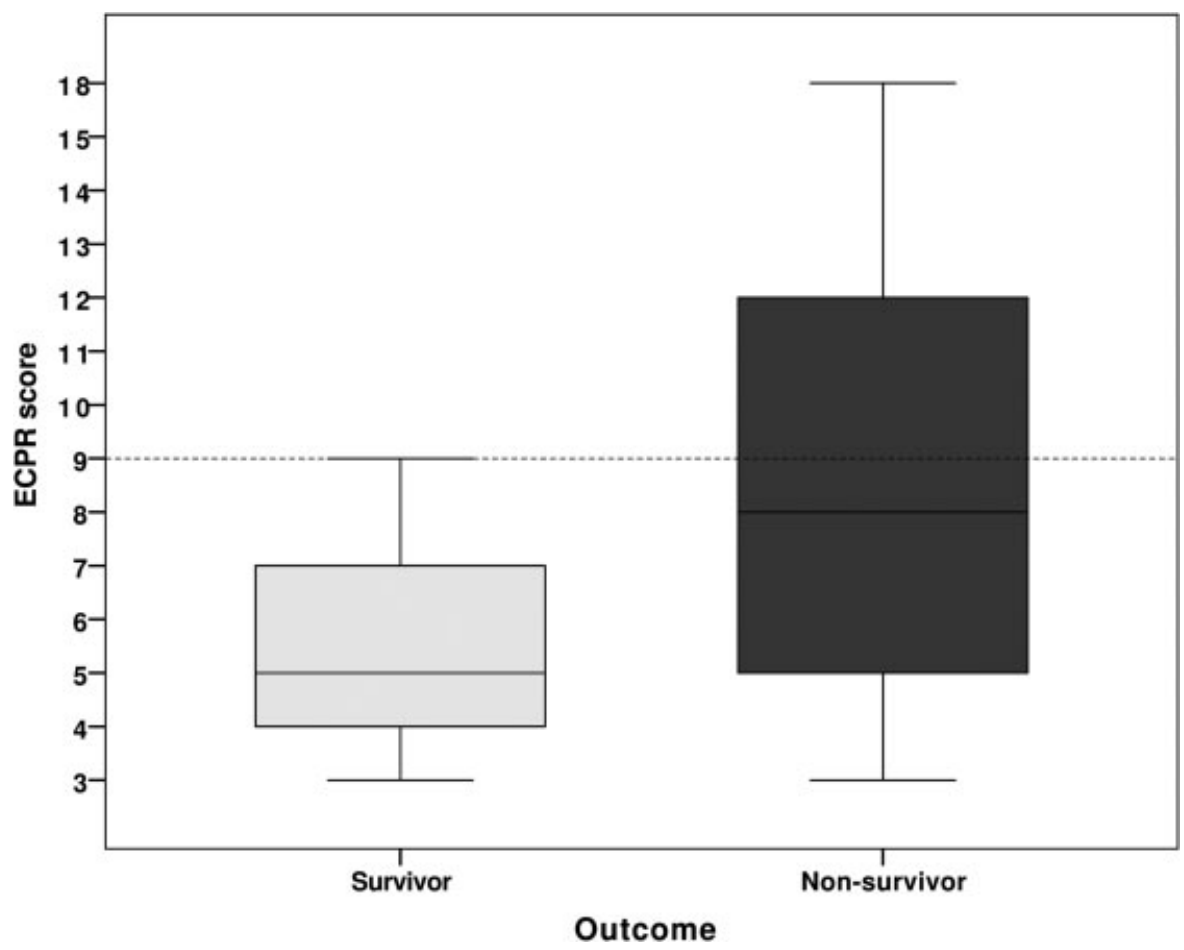

Fig. 2 Boxplot of the extracorporeal life support during active resuscitation score, in the patients who survived (light gray) and who died (dark gray); $p=0.01$. The horizontal dashed line represented the cutoff value of 9 points, which had a positive predictive value of $94 \%$ ( $95 \%$ confidence interval: 71-99). 
ECMO team, resulting in potentially long durations of CPR. It needs to be considered that if the benefits do not outweigh the negative side effects for a particular treatment/therapy, then it might be in the patient's best interest to withhold that treatment/therapy. ${ }^{27}$ Furthermore, in the setting of limited resources and the ethical principle of resource equality, the attending physician has the duty to reasonably allocate costly and potential futile treatment options.

This study is subject to various limitations, mostly inherent to retrospective observational series. First, due to small sample size multivariate analyses were not possible. Then, as there is no formal ECPR recommendation in place, the decision to escalate level of care to ECMO was left to the attending physician, and there was no control group which benefited from CPR only. Additionally, our survival rates were lower than those of the ELSO registry, which could limit the generalization of our results. Our results could be explained in part by our ECPR organization without in-house cardiac surgery and perfusionist teams during nights and weekends. Furthermore, the variables we collected cannot entirely describe the complex pre-ECMO situation, which might have led to some bias. However, the variables that were collected are similar to those reported in other studies. Moreover, as we focused on pre-ECMO variables, we did not report ECMO complication, which might have been associated with clinical outcome. Afterward, regarding demographic data, we did not collect whether the patient was admitted through the emergency department (out-of-hospital cardiac arrest [OHCA]) or was already admitted in the hospital (in-hospital cardiac arrest). This location factor might influence our results, although CPR duration reflects OHCA and last $\mathrm{pH} /$ lactate before cannulation is a surrogate marker for CPR quality. Also, the exact delay between the last blood gas and the initiation of ECMO was not recorded. However, the concept of "last gas prior to ECMO initiation" is pragmatic and therefore strengthens the generalizability of our results. Furthermore, because of the retrospective design, we were not able to assess the CPR quality. Besides, although there might have been some changes in technology and management protocols over time, this did not influence the outcome in our series. Finally, like most pediatric ECPR studies, we were not able to systematically assess both nonfocal and long-term neurological outcomes.

\section{Conclusion}

The identification of appropriate ECPR candidates remains an ongoing challenge for all the ECPR centers and implies integration of patient-related factors that can predict mortality. In our population, a score based on three variables, that is, length of $\mathrm{CPR}$, last $\mathrm{pH}$, and last lactate, easily available prior to ECLS initiation, had a good discrimination and could appropriately predict mortality. This score now needs to be evaluated in a larger population.

\section{Conflict of Interest}

None declared.

\section{Acknowledgments}

We would like to thank Alexandrine Brunner and Natacha Dubois for the help in collecting the data. We would also like to thank Grace Henderson for help with the manuscript.

\section{References}

1 del Nido PJ, Dalton HJ, Thompson AE, Siewers RD. Extracorporeal membrane oxygenator rescue in children during cardiac arrest after cardiac surgery. Circulation 1992;86(5, Suppl): II300-II304

2 Lasa JJ, Rogers RS, Localio R, et al. Extracorporeal cardiopulmonary resuscitation (E-CPR) during pediatric in-hospital cardiopulmonary arrest is associated with improved survival to discharge: a report from the American Heart Association's Get with the Guidelines-Resuscitation (GWTG-R) Registry. Circulation 2016; 133(02):165-176

3 Barbaro RP, Paden ML, Guner YS, et al; ELSO member centers. Pediatric Extracorporeal Life Support Organization Registry International Report 2016. ASAIO J 2017;63(04):456-463

4 Maconochie IK, de Caen AR, Aickin R, et al; Pediatric Basic Life Support and Pediatric Advanced Life Support Chapter Collaborators. Part 6: Pediatric basic life support and pediatric advanced life support: 2015 International Consensus on Cardiopulmonary Resuscitation and Emergency Cardiovascular Care Science with Treatment Recommendations. Resuscitation 2015;95:e147-e168

5 Joffe AR, Lequier L, Robertson CM. Pediatric outcomes after extracorporeal membrane oxygenation for cardiac disease and for cardiac arrest: a review. ASAIO J 2012;58(04):297-310

6 Fiser DH. Assessing the outcome of pediatric intensive care. J Pediatr 1992;121(01):68-74

7 Shann F. Are we doing a good job: PRISM, PIM and all that. Intensive Care Med 2002;28(02):105-107

8 Keegan MT, Gajic O, Afessa B. Severity of illness scoring systems in the intensive care unit. Crit Care Med 2011;39(01):163-169

9 Alsoufi B, Awan A, Manlhiot C, et al. Results of rapid-response extracorporeal cardiopulmonary resuscitation in children with refractory cardiac arrest following cardiac surgery. Eur J Cardiothorac Surg 2014;45(02):268-275

10 Chan T, Thiagarajan RR, Frank D, Bratton SL. Survival after extracorporeal cardiopulmonary resuscitation in infants and children with heart disease. J Thorac Cardiovasc Surg 2008; 136(04):984-992

11 Thiagarajan RR, Laussen PC, Rycus PT, Bartlett RH, Bratton SL. Extracorporeal membrane oxygenation to aid cardiopulmonary resuscitation in infants and children. Circulation 2007;116(15): 1693-1700

12 Debaty G, Babaz V, Durand M, et al. Prognostic factors for extracorporeal cardiopulmonary resuscitation recipients following out-of-hospital refractory cardiac arrest. A systematic review and meta-analysis. Resuscitation 2017;112:1-10

13 Wolf MJ, Kanter KR, Kirshbom PM, Kogon BE, Wagoner SF. Extracorporeal cardiopulmonary resuscitation for pediatric cardiac patients. Ann Thorac Surg 2012;94(03):874-879, discussion 879-880

14 Kane DA, Thiagarajan RR, Wypij D, et al. Rapid-response extracorporeal membrane oxygenation to support cardiopulmonary resuscitation in children with cardiac disease. Circulation 2010; 122(11, Suppl):S241-S248

15 Huang SC, Wu ET, Chen YS, et al. Extracorporeal membrane oxygenation rescue for cardiopulmonary resuscitation in pediatric patients. Crit Care Med 2008;36(05):1607-1613

16 Allan CK, Thiagarajan RR, Armsby LR, del Nido PJ, Laussen PC. Emergent use of extracorporeal membrane oxygenation during pediatric cardiac catheterization. Pediatr Crit Care Med 2006;7 (03):212-219 
17 Prodhan P, Fiser RT, Dyamenahalli U, et al. Outcomes after extracorporeal cardiopulmonary resuscitation (ECPR) following refractory pediatric cardiac arrest in the intensive care unit. Resuscitation 2009;80(10):1124-1129

18 Morris MC, Wernovsky G, Nadkarni VM. Survival outcomes after extracorporeal cardiopulmonary resuscitation instituted during active chest compressions following refractory in-hospital pediatric cardiac arrest. Pediatr Crit Care Med 2004;5(05):440-446

19 Kelly RB, Porter PA, Meier AH, Myers JL, Thomas NJ. Duration of cardiopulmonary resuscitation before extracorporeal rescue: how long is not long enough? ASAIO J 2005;51(05):665-667

20 Matos RI, Watson RS, Nadkarni VM, et al; American Heart Association's Get With The Guidelines-Resuscitation (Formerly the National Registry of Cardiopulmonary Resuscitation) Investigators. Duration of cardiopulmonary resuscitation and illness category impact survival and neurologic outcomes for in-hospital pediatric cardiac arrests. Circulation 2013;127(04):442-451

21 Tajik M, Cardarelli MG. Extracorporeal membrane oxygenation after cardiac arrest in children: what do we know? Eur J Cardiothorac Surg 2008;33(03):409-417
22 Wengenmayer T, Rombach S, Ramshorn F, et al. Influence of lowflow time on survival after extracorporeal cardiopulmonary resuscitation (eCPR). Crit Care 2017;21(01):157

23 Delmo Walter EM, Alexi-Meskishvili V, Huebler M, et al. Rescue extracorporeal membrane oxygenation in children with refractory cardiac arrest. Interact Cardiovasc Thorac Surg 2011;12(06):929-934

24 Cardarelli MG, Young AJ, Griffith B. Use of extracorporeal membrane oxygenation for adults in cardiac arrest (E-CPR): a metaanalysis of observational studies. ASAIO J 2009;55(06):581-586

25 Aharon AS, Drinkwater DC Jr, Churchwell KB, et al. Extracorporeal membrane oxygenation in children after repair of congenital cardiac lesions. Ann Thorac Surg 2001;72(06):2095-2101, discussion 2101-2102

26 Extracorporeal Life Support Organization. ECMO Outcome Prediction Scores for Estimates of Likelihood of Survival. Available at: https://www.elso.org/Resources/ECMOOutcomePredictionScores.aspx. Accessed April 9, 2018

27 Welie JV, Ten Have HA. The ethics of forgoing life-sustaining treatment: theoretical considerations and clinical decision making. Multidiscip Respir Med 2014;9(01):14 